\title{
Systemic Sclerosis and the
}

\section{Gastrointestinal Tract-Clinical}

\section{Approach}

\author{
Yolanda Braun-Moscovici, M.D. ${ }^{1,2 *}$, Rita Brun, M.D. ${ }^{2,3}$ and Marius Braun, M.D. ${ }^{4,5}$ \\ 'B. Shine Rheumatology Unit, Rambam Health Care Campus, Haifa, Israel; ${ }^{2 R a p p a p o r t ~ F a c u l t y ~ o f ~}$ \\ Medicine, Technion-Israel Institute of Technology, Haifa, Israel; 'Department of Gastroenterology, \\ Rambam Health Care Campus, Haifa, Israel; "Liver Institute, Beilinson Hospital, Petach Tiqwa, Israel; \\ and ${ }^{5}$ Sackler School of Medicine, Tel Aviv University, Tel Aviv, Israel
}

\begin{abstract}
ABST RACT
Sy stemic sclerosis (SSc) is a multisystem disease characterized by functional and structural abnormalities of small blood vessels, fibrosis of the skin and internal organs, immune system activation, and autoimmunity. The gastrointestinal tract is involved in nearly all patients and is a source of significant morbidity andeven mortality. The aim of this review is to summarize the pathogenesis and to provide a clinical approach to these patients.
\end{abstract}

KEY WORDS: Gastric antral vascular ectasia, gastrointestinal tract involvement, systemic sclerosis

\section{INT RODUCTION}

The first case of scleroderma was diagnosed by Hippocrates in the fourth century $\mathrm{BC}$ in a patient with "thick skin." ${ }^{1}$ More than two thousand years later, in 1836, Giovambattista Fantonetti coined the

\begin{abstract}
Abbreviations: A PC, argon plasm a coagulation; GAVE, gastric antral vascular ectasia; GERD, gastroesophageal r eflux disea se; GI, g astrointestinal; GIT, g astrointestinal tract; LES, lower esophageal sphincter; PCI, pneumatosis cystoides in testinalis; SIBO, sm all intestinal bacterial ov ergrowth; SSc, sy stemic sclerosis; VCE, video capsule endoscopy .

Citation: Br aun-MoscoviciY, Braun R, Braun M. Sy stem ic Sclerosis and the Gastrointestinal Tract-Clinical Approach. Ram bam Maimonides Med J 2016;7 (4):eo031. doi:10.5041/RMMJ.10258 Review

Copy right: (C) 2016 Braun-Moscovici et al. This is an open-access a rticle. All its content, except where otherwise noted, is distributed under the terms of the Creative Com mons Attribution License (http://creative com mons.org/licenses/by/3.0), wh ich permits unrestricted $\mathrm{u}$ se, distribution, and reproduction in any m edium, provided the original work is properly cited.
\end{abstract}

Conflict of interest: No pot ential conflict of in terest relevant to this article was reported.

* Tow hom correspondence sh ould be a ddressed. E-mail: y_braun@rambam.health.gov.il 
term "scleroderma," which is derived from Greek terminology "skleros" (hard) and "derma" (skin). ${ }^{1}$ The term "systemic sclerosis" was conceived by Robert H. Goetz, a heart surgeon, in 1945, who described scleroderma as a disease that infiltrates several internal organs. ${ }^{2}$

\section{PAT HOGENESIS}

Systemic sclerosis (SSc) is a multisy stem disease characterized by functional and structural abnormalities of small blood vessels, fibrosis of the skin and internal organs, immune system activation, and autoimmunity. The cause of SSc is unknown. An integrated hypothesis of the pathogenesis of SSc includes a combination of abnormalities in the vascular and in the immune systems on a background of genetic susceptibility and in the presence of environmental stimuli, which leads to further augmentation of the immune sy stem's activation and, ultimately, to fibroblast proliferation, collagen deposition, and destruction of normal tissue architecture. 3

The vascular hypothesis suggests that the primary event in SSc occurs at the level of capillaries and small vessels and manifests as endothelial cell injury and activation. Vascular pathology is characterized by abnormalvasoreactivity, dysregulationof vasoconstrictive molecules and their receptors, upregulation of intracellular signaling kinases, altered balance of hypoxia-induced vasculargrowth factors, and aberrantfunction of vascular cells and autoimmune effector cells, which all lead to insufficient neoangiogenesis. $4^{-10}$

During the last decade, studies have emphasized the role of the innate and the adaptive immune system in the pathogenesis of SSc. Genome-wide approaches have revealed that increased expression of genes associated with SSc susceptibility and/or disease phenotype plays a major role in the regulation of the immune system. T cells, fibroblasts, growth factors, chemokines, and endothelin- 1 are all key factors in disease pathophysiology .11-16

Systemic sclerosis has been classified according to the extent of clinically detectable skin tightness into limited cutaneous SSc (hardening confined to skin from elbows distally and from knees distally) and diffuse cutaneous SSc (hardening of skin including proximal extremities and the trunk). ${ }^{17}$ Both forms involve the internal organs.

Involvement of the gastrointestinal tract (GIT) in SSc is extremely frequent; it is a leading cause of morbidity and the third most common cause of mortality in this disease. Esophageal abnormalities occur in up to $90 \%$ of patients, stomachinvolvement can be documented in $50 \%$ or more of patients, and small bowel, colonic, and anorectal involvement occur in $50 \%-70 \%$ of SSc patients. ${ }^{18-20}$

The pathogenesis of GIT involvement is thought to include early vascular damage to the vasa nervorum of the nerves innervating the GIT. This leads to neurological dy sfunction, particularly involving autonomic pathways. ${ }^{21,22}$ The activation of the immune sy stem may contribute to neurologicaldysfunction by production of antibodies which specifically inhibit M3-muscarinic receptor-mediated enteric cholinergic neurotransmission. ${ }^{23}$ Endothelial/lymphocyte activation leads to prominent infiltration of CD4 + T ly mphocytes as well as CD2O + B ly mphocytes into the gastric mucosa of patients with SSc and perhaps represents an early event in gastrointestinal (GI) pathology. 24 With damage to innervation, smooth muscle atrophies and is eventually replaced by fibrotic tissue. With increasing atrophy and tissue replacement, the GIT becomes progressively less effective and less responsive to therapeutic agents. 25

\section{SYMPT OMSAND SIGNS}

Motility disorders and vascular mucosal lesions are the main manifestations of GIT involvement in SSc. The entire GIT may be involved from the mouth to anus in both limited and diffuse SSc.

Oral cavity abnormalities are common in SSc. Tightening of the perioral skin secondary to fibrosis may cause severe feeding impairment. Xerostomia due to Sicca sy ndrome may occur in $14 \%-20.5 \%$ of SSc patients and may further decrease oral intake. ${ }^{26}$

Esophageal involvement is the most frequent gastrointestinal manifestation of SSc and occurs in up to $90 \%$ of patients. Multiple abnormalities of esophageal function cause the clinical manifestations of severe gastroesophageal reflux and dysphagia to liquids and solids. The hallmark of SSc in the esophageal body is ineffective esophageal motility with low or absent contractile activity. Subsequently, the lower esophageal sphincter (LES) is hy potensive, and hiatal hernia is common, resulting in almost free regurgitation of gastric acidic contentsinto the esophagus. In addition, saliva and esophageal mucosal secretions production is reduced. Heartburn and dysphagia are the most common complaints. Hoarseness, aty pical chest 
pain, nocturnal cough, and regurgitation may also occur.

Ineffective motility, hypotensive LES, poor acid and bolus clearance, and lack of buffer secretions all contribute to esophageal mucosal damagesecondary to refractory acid reflux. Late complications include esophageal stenosis, strictures, and, ultimately, Barrett's esophagus and intestinal metaplasia. ${ }^{19}$ The prevalence of Barret's esophagus in SSc was found to be $12.7 \%$, similar to the prevalence in patients with gastroesophageal reflux disease (GERD). ${ }^{27} \mathrm{An}$ increased risk of esophageal adenocarcinoma was reported in SSc patients and was associated with the occurrence of dy splasia in Barrett's esophagus. ${ }^{28}$

Gastroesophageal reflux disease wassuggested to be a risk factor for the development of interstitial lung disease. ${ }^{29}$

Gastroparesis is common in SSc patients, but its true prevalence is unknown. It is important to be aware of diagnosis and actively look for it, as its appropriate management can relieve the patient's symptoms significantly.

Early satiety, postprandial fullness, nausea and vomiting, regurgitation of gastric contents, abdominal pain, and, in severe cases, malnutrition due to inability to maintain adequate oral intake are the clinical manifestation of gastroparesis.

Autonomic dy sfunction plays an important role in the pathogenesis of this dy smotility. ${ }^{21}$

Small bowel involvement has been reported in $50 \%-70 \%$ of SSc patients ${ }^{18-20}$ and may lead to high morbidity and life-threatening complications, such as severe malabsorption and pseudo-obstruction. Small bowel hy pomotility induces stasis of intestinal contents and small intestinal bacterial overgrowth (SIBO), which contribute to bloating, abdominal pain, nausea, vomiting, diarrhea, malabsorption, and weight loss. $3^{\circ}$ The prevalence of SIBO in SSc has been reported to be 30\%-62\%.31-35 Small intestinal bacterial overgrowth is one of the main pathogenetic factors of malabsorption which is associated with $50 \%$ mortality over 8.5 yearsin SSc patients. ${ }^{6}$ Rare or absent motor migratory complexes (MMCs), which serve as a house-keeping mechanism of intestines, contribute to SIBO, while decreased postprandial contractility of the small intestine is one of the causes of postprandial pain and discomfort. Clinically, diarrhea, bloating, and nutritional deficiencies due to malabsorption should raise the suspicion of SIBO.

Intestinal pseudo-obstruction is a rare cause of hospitalization in patients with SSc, but is associated with high in-hospital mortality. 37

Pneumatosis cystoides intestinalis (PCI) is a rare complication of SSc and is considered a sign of poor prognosis. 38,39 It is characterized by development of multiple intramural air-filled cysts, due to anaerobic bacterial overgrowth in the intestine and increased intraluminal hy drogen production. The cysts may rupture and cause pneumoperitoneum and secondary peritonitis. The risk of perforation is already increased in SSc patients due to fibrosis and loss of compliance of the intestinal wall. 40

The colon is frequently involved in SSc, although it is not always sy mptomatic. Abnormal motility pattern has been found in $75 \%$ of asy mptomatic SSc patients. 41

Constipation and fecal incontinence due to reduced colonic motility and hypotensive anal sphincter are the main issues involving the colon. ${ }^{42}$ Fecal incontinence is an under-reported but frequent complication of SSc. Patients withdiarrhea are especially prone to incontinence episodes.

Malnutrition and weight loss result from the multiple anatomic and functional abnormalities through the whole gastrointestinal tract in SSc, but studies assessing their prevalence are lacking.

Vascular lesions of the mucosa may cause severe anemia in SSc patients. The lesions may bescattered throughout the entire intestine or may involve only the stomach antrum (gastric antral vascular ectasia). 43 Gastric antral vascular ectasia (GAVE) is characterized by a pathognomonic endoscopic pattern, mainly represented by red spots either organized in stripes radially originating at the pylorus ("watermelon stomach") or arranged diffusely ("honeycomb stomach"). 44 "Watermelon stomach" is the "classic" and more familiar form of GAVE. There are conflicting data regarding the prevalence of GAVEin SSc. Previous studies estimated a $1 \%-5.7 \%$ prevalence of GAVE in SSc patients. 45,46 On the other hand, a study performed in patients with early diffuse SSc reported a much higher prevalence of GAVE: $22.3 \% .47$ A recent study, using video capsule endoscopy (VCE), found evidence of "watermelon stomach" in $18 \%$ of SSc patients. 48 


\section{CLINICAL APPROACH AND ASSESSMENT TOOLS}

In daily practice, the patient presents with a mixed clinical picture of refractory GERD, diarrhea, bloating, dy sphagia, weight loss, and nutritional deficiencies. The diagnostic studies should be directed to identify the GI site involved, assess severity, and rule out other etiologies. Sy stematic evaluation of motoric function of the GI tract in SSc patients enables the clinician to build an appropriate treatment plan for the individual patient.

Anamnesis should be directed to identify the most bothersome symptoms of the patient. First, nutritional status should be evaluated, as often weight loss is a sensitive sign of poor functional status of the GI tract. Questions regarding symptoms of gastroparesis and fecalincontinence should be activ ely asked, because patients may have difficulties in sharing this information.

There is no single objective measure to assess the extent and severity of GI involvement in SSc patients.

Upper gastrointestinal (UGI) endoscopy is the gold standard for esophagus and stomach morphology assessment. The procedure is a means for visualizing tissue, for sampling, and for therapeutic interventions (e.g. in cases of bleeding from GAVE). Standard endoscopic imaging is useful for the detection of grossly visible lesions but may be less sensitive for the detection of early or subtle mucosal changes.

Gastroscopy is the gold standard for diagnosis of GAVE and for assessment of its severity. Gastric biopsy can help to diagnose the condition in equivocal cases. The histological pattern, although not pathognomonic, is characterized by the co-presence of ectasia and/or fibrin thro mbi, spindle cell proliferation, and fibrohyalinosis. Gastric antral vascular ectasia can also be treated during UGI endoscopy using argon plasma coagulation.

In addition, information about the functional motility status of the upper GI tract can be obtained during UGI endoscopy: esophageal and gastric contents despite fasting, dilated esophagus, widely opened gastroesophageal junction, hiatal hernia, and lack of peristalsis are highly suggestive of hypomotility.

Evaluation of motor GI function should be performed in patients with symptoms suggesting motility abnormalities. High-resolution esophageal manometry is a new technique to evaluate esophageal motility. Reflux extent and severity, as well as a response to acid suppression medications, is studied using esophageal reflux monitoring with $\mathrm{pH}$ or $\mathrm{pH} /$ impedance probes based on intranasalcatheter, or, recently, the more comfortable wireless Bravo pH-metry capsule.

Gastric emptying can be assessed by gastric scintigraphy or breath test.

Small intestinal bacterial overgrowth is diagnosed by breaths tests, which have multiple limitations, or, rarely performed clinically, by culture of jejunal aspirate. $49 \mathrm{~A}$ more practical approach would be empirically treating SIBO with antibiotics and a retrospective diagnosis based on clinical response. $5^{0}$

Colonic transit time can be non-invasively measured using the SITZMARKS test (ingestion of a capsule containing 24 radiop aque markers that are visible throughout the digestive tract via X-ray).

Anorectal function is studied by anorectal manometry, preferably using high-resolution technology, which provides information of thefunctional status of the sphincter. Transrectal ultrasound(US) can be used to visualize the anatomy of internal and external anal sphincters. Magnetic resonance imaging has been used for evaluation of anorectal anatomy in SSc patients, but it is much more expensive and without clear advantages in this patient group compared to US. ${ }^{1}$

Video capsule endoscopy (VCE) identifies a high prevalence of gastrointestinal mucosal abnormalities, especially potentially bleedingvascular mucosal lesions (watermelon stomach, gastric and/or small intestinal telangiectasia, gastric and/or small intestinal angiodysplasia). ${ }^{48} \mathrm{Hy}$ pomotility problems in SSc may raise concern regarding the use of VCE in these patients.

\section{T REATMENT}

To date, the management of GIT inv olvement in SSc remains empirical and symptom-driven. The ultimate goal of a systematic complex approach to GI abnormalities in scleroderma patient is the improvement of their nutritional status and quality of life.

High-dose, twice daily acid suppression treatment with a proton pump inhibitor is a mainstay therapy for GERD. Sometimes a combination with 
H2 blockers (ranitidine, famotidine) is needed to control the night-time breakthrough acidreflux. It is important to notice that poorly controlled GERD in SSc patients, despite optimal medical treatment, is often secondary to gastroparesis, and specific measures to improve gastric emptying should be incorporated into the treatment plan.

Treatment of gastroparesis starts with nutritional intervention-multiple small meals and low-fiber diet. When needed, pro-kinetic medications (domperidone, metoclopramide, erythromycin) are added to improve gastric emptying.

Small intestinal bacterial overgrowth is usually treated with antibiotics. Recently, rifaximin has been used for treating SIBO, with the advantages of being a non-absorbable antibiotic with few systemic side effects, as well as possible positive influenceson intestinal flora. $5^{2}$ Octreotide has been reported to be helpful in selected cases, especially in patients with recurrent pseudo-obstruction of the small bowel. ${ }^{41,53}$

Fecal incontinence requires a complex approach, combining medical and nutritional interventions with phy sical therapy, preferably anorectal biofeedback training. Unfortunately, the response rate to these therapies is not usually satisfactory in SSc patients. New therapies for fecal incontinence, such as sacral nerve stimulation, have been reported to be unsuccessful in scleroderma. 54

Nutritional deficiencies should be corrected (vitamins B12, D, etc.). Deficiency of vitamin B12 is common and should be treated. In case of low BMI an appropriate nutritional plan shouldbe developed for each patient. Patients with chronic intestinal pseudo-obstruction who cannot tolerate enteral feeding may need prolonged total parenteral nutrition. 55,56

Some studies suggest probiotics may be useful for treatment of SSc-associated distention and bloating, but the small number of patients and the diversity of probiotics used do not permit any consistent treatment recommendation. 57

Gastric antral vascular ectasia should be treated by endotherapy-argon plasma coagulation (APC). Treatment with APC can reduce the need for blood transfusions. ${ }^{6}$ Most patients with early diffuse SSc and GAVE will need recurrent endoscopic coagulations to overcome UGI bleeding. In these patients with early diffuse progressive disease, concomitant immune suppression with cyclophosphamide or mycophenolate mofetil mightcontribute to significant improvement and eventually to final resolution of the UGI bleeding. 58-60 and our unpublished data

Data regarding the influence of immunomodulatory therapy on GIT involvement are scarce. A recently published study reported a beneficial effect of long-term therapy with intravenous immunoglobulins on some of the GI manifestations in patients with overlap of SSc and myositis. ${ }^{61}$ There are no data about the influence on GIT in SSc patients without myositis.

\section{CONCLUSIONS}

The gastrointestinal tract is one of the main systems involved in SSc patients causing significant morbidity and ev en mortality. There is no single objective measure to assess the extent and severity of GI involvement in SSc patients. A multidisciplinary approach with a rheumatologist, gastroenterologist, and sometimes a nutritionist is mandatory in all patients with severe gastrointestinal involvement. The management of GIT involvement in SSc remains empirical and symptom-driven. Data regarding the influence of immunomodulatory therapy on GIT involvement are scarce. Welldesigned and high-powered prospective studies are needed to determine the effect of immunosuppressive treatment on the onset of GI tract disease, especially in early SSc.

\section{REFERENCES}

1. Coy le W. A brief history of scleroderma. Scleroderma News 1988;8:2.

2. Konstantinov IE. Robert H. Goetz: thesurgeon who performed the first successful clinical coronary artery by pass operation. Ann Thorac Surg 2 000;69:196672. Full Text

3. Furst DE, Clements PJ. Pathogenesis, Fu sion (Summary). In: Clements PJ, Furst DE, eds. Sy stemic Sclerosis. Baltimore: Williams \& Wilkins; 1996:275-86.

4. Muller-Ladner U, Distler O, Ibba-Manneschi L, Neumann E, Gay S. Mechanisms of v ascular damage in sy stemic sclerosis. Autoimmunity $2009 ; 42: 587-$ 95. Full Text

5. Kuwana M, Okazaki Y, Yasuoka H, Kawakami Y, Ikeda Y. Defective vasculogenesis in systemic sclerosis. Lancet 2004;364:603-10. Full Text

6. Distler O, Distler JH, Scheid A, et al. Uncontrolled expression of vascular en dothelial growth factor and its receptors leads to in sufficient skin angiogenesis in 
patients with sy stemic sclerosis. Circ Res 2004; 95:109-16. Full Text

7. Distler O, Del Rosso A, Giacomelli R, et al. Angiogenic and angiostatic factors in sy stem ic sclerosis: increased levels of vascular endothelial growth factor are a feature of the earliest disease stages and are associated with the absence of fingertip ulcers. Arthritis Res 2002;4:R11. Full Text

8. Trojanowska M. Role of PDGF in fibrotic diseases and systemic sclerosis. Rheumatology (Oxford)2008; 47(Suppl 5):v 2-4. Full Text

9. Distler JH, Wenger RH, Gassmann M, et al. Physiologic responses to hy poxia and implications for hy poxia-inducible factors in the pathogenesis of rheumatoid arthritis. Arthritis Rheum 2004;50:1023. Full Text

10. From mer K, Muller-Ladner U. Expression and function of ETA andETB receptors in SSc. Rheumatology (Oxford) 2008;47 (Suppl 5):v 27-8. Full Text

11. Denton CP, Abraham DJ. Transforming growth factor-beta and con nective tissu egrowth factor: key cy tokines in scleroderma pathogenesis. Curr Opin Rheumatol 2001;13:505-11. Full Text

12. Ong VH, Evans LA, Shiwen X, et al. Monocyte chem oattractant protein 3 as a mediator of fibrosis: ov er expression in systemic sclerosis and the ty pe 1 tight-skin mouse. Arthritis Rheum2003;48:1979-91. Full Text

13. Mayes MD. Endothelin and endothelin receptor antagonists in systemic rheumatic disease. Arthritis Rheum 2003;48:1190-9. Full Text

14. Michels-van Amelsfort JMR, Walter GJ, Taams LS. $\mathrm{CD} 4+\mathrm{CD} 25+$ regulatory $\mathrm{T}$ cells in systemic sclerosis and other rheumatic diseases. Expert Rev Clin Immunol 2011;7:499-514. Full Text

15. Usategui A, del Rey MJ, Pablos JL. Fibroblast abnormalities in the pathogenesis of systemic sclerosis. Expert Rev Clin Immunol 2011;7:491-8. Full Text

16. York MR. Novel insights on the role of the innate im m une system in systemic sclerosis. Expert Rev Clin Immunol 2011;7:481-9. Full Text

17. LeRoy EC. Scleroderma (systemic sclerosis): classification, subsets and pathogenesis. J Rheumatol 1988; 15:202-5.

18. Sjogren RW. Gastrointestinal motility disorders in scleroderma. Arthritis Rheum 1994;37:1265-82. Full Text

19. Sjogren RW. Gastrointestinal features of scleroderma. Curr Opin Rheumatol 1996;8:569-75. Full $\underline{\text { Text }}$
20. Lock G, Holstege A, Lang B, Scholmerich J. Gastrointestinal manifestations of progressive sy stemic sclerosis. Am J Gastroenterol 1997;92:763-71.

21. Iovino $\mathrm{P}$, Valentini $\mathrm{G}$, Ciacci $\mathrm{C}$, et al. Proximal stom ach function in systemic sclerosis. Relationship with auton omic nerve function. Dig Dis Sci2001;46: 723-30. Full Text

22. Cohen S, Risher R, LipshutzW, Turner R, My ers A, Schumacher R. The pathogenesis of esophageal dy sfunction in scleroderma and Raynaud's disease.J Clin Invest 1972;51:2663-8. Full Text

23. Goldblatt F, Gordon TP, Waterman SA. Antibody mediated gastrointestinal dy smotility in scleroderma. Gastroenterol 2002;123:1144-50. Full Text

24. Manetti M, Neumann E, Muller A, et al. Endothelial/ ly mphocyte activation leads to prominent CD4 $+\mathrm{T}$ cell infiltration in the gastric mucosa of patients with sy stemic sclerosis. Arthritis Rheum 2008;58:286673. Full Text

25. Greydanus MP, Camilleri M. Abnormal postcibal antral and small bowel motility dueto neuropathy or myopathy in systemic sclerosis. Gastroenterology 1989;96:110-15. Full Text

26. Avouac J, Sordet C, Depinay C, et al. Sy stemic scler osis-a ssociated Sjögren's sy ndrome and relationship to the limited cutaneous subty pe: results of a prospective study of sicca syndrome in 133 con secutiv epatients. Arthritis Rheum 2006;54:2243-9. Full $\underline{\text { Text }}$

27. Wipff J, Allanore Y, Soussi F, et al. Prevalence of Barrett's esophagus in systemic sclerosis. Arthritis Rheum 2005;52:2882-8. Full Text

28. Wipff J, Coriat R, Masciocchi M, et al. Outcomes of Barrett's oesophagus related to sy stemic sclerosis: a 3-y ear EULAR Scleroderma Trials and Research prospective follow-up study. Rheumatology (Oxford) $2011 ; 50: 1440-4$. Full Text

29. Gyger G, Baron M. Sy stemic sclerosis: gastrointestinal disease and its management. Rheum Dis Clin North Am 2015;41:459-73. Full Text

30. Abu-Shakra M, Guillemin F, Lee P. Gastrointestinal manifestation s of systemic sclerosis. Semin Arthritis Rheum 1994;24:29-39. Full Text

31. Cobden I, Axon AT, Ghoneim AT, McGoldrick J, Rowell NR. Small intestinal bacterial growth in sy stemic sclerosis. Clin Exp Dermatol 1980;5:37-42. Full Text

32. Kay e SA, Lim SG, Taylor M, Patel S, Gillespie S, Black CM. Small bowel bacterial ov ergrowth in sy stemic scler osis: detection using direct and indirect methods and treatment outcome. Br J Rheumatol 1995;34: 265-9. Full Text 
33. Gasbarrini A, Corazza GR, Gasbarrini G, et al. Methodology and indications of $\mathrm{H} 2$-breath testing in gastrointestinal diseases: the Rome Consensus Conference. Aliment Ph armacol Ther 2009;29(Suppl 1):1-49. Full Text

34. Owy ang C. Octreotide in gastrointestinal m otility disorders. Gut 1994;35(Suppl):S11-14. Full Text

35. King CE, Toskes PP. Comparison of the 1 -gram [14C]xylose, 10-gramlactulose-H2, and 80-gram glucose-H2 breath tests in patients with small intestinebacterial ov ergrowth. Gastroenterology 1986;91:1447-51. Full Text

36. Jaov isidha K, Csuka ME, Almagro UA, Soergel KH. Sev ere gastrointestinal involv em ent in sy stemic sclerosis: report of five cases and review of the literature. Semin Arthritis Rheum 2008;34:689-702. Full Text

37. Valenzuela A, Li S, Becker L, et al. Intestinal pseu doobstruction in patients with systemic sclerosis: an analy sis of the Nationwide Inpatient Sample. Rheum atology (Oxford) 2016;55:654-8. Full Text

38. Balbir-Gurman A, Brook OR, Chermesh I, BraunMoscovici Y. Pneumatosis cystoides intestinalis in scleroderma-related conditions. Intern Med J 2012; 42:323-9. Full Text

39. Quiroz ES, Flannery MT, Martinez EJ, Warner EA. Pneu matosis cystoides intestinalis in progressive sy stemic sclerosis: a case report and literature review. Am J Med Sci 1995;310:252-5.

40. Ebert EC, Ruggiero FM, Seibold JR. Intestinal perfor ation: a common complication of scleroderma. Dig Dis Sci 1997;42:549-53. Full Text

41. Ebert EC. Gastric and enteric involvement in progressive systemic sclerosis. J Clin Gastroenterol 2008;42:5-12. Full Text

42. Umar SB, Griffing L, Garcia H, Foxx-Orenstein AE, DiBaise JK, Crowell MD. Theimpact of pelvic floor and lower gastrointestinal symptoms on quality of life in women with systemic sclerosis. J Clin Gastroenterol 2016;50:e55-9. Full Text

43. Fu ccio L, Mussetto A, Laterza L Eusebi LH, Bazzoli F. Diagnosis and management of gastric antral vascular ectasia. World J Gastrointest Endosc 2 013;5:6-13. Full Text

44. Ito M, Uchida Y, Kamano S, Kawabata H, Nishioka M. Clinical comparisons between two subsets of gastric antralvascular ectasia. Gastrointest Endosc 2001;53:764-70. Full Text

45. Ghrénassia E, AvouacJ, Khanna D, et al. Prevalence, correlates and ou tcomes of gastric a ntral v ascular ecta sia in systemic sclerosis: a EUSTAR case-control study. J Rheumatol 2014;41:99-105. Full Text
46. Marie I, DucrotteP, Antonietti M, et al. Watermelon stomach in systemic sclerosis: its incidence and management. Aliment Pharm acol Ther 2008;2 8: 412-21. Full Text

47. Hung EW, Mayes MD, Sharif R, et al. Gastric antral $\mathrm{v}$ a scular ectasia and its clinical cor relates in patients with early diffuse sy stemic sclerosis in the SCOT trial. J Rheumatol. 2013;40:455-60. Full Text

48. Marie I, Antonietti M, Houivet E, et al. Gastrointestinal mucosal abnormalities u singvideocapsule endoscopy in systemic sclerosis. Aliment Pharmacol Ther 2014;40:189-99. Full Text

49. Braun-Moscovici Y, Braun M, Khanna D, BalbirGurman A, Furst DE. What tests should y ou use to assess small intestinal bacterial overgrowth in sy stemic sclerosis? Clin Exp Rheumatol 2015;33 (4 Suppl 91):S117-22.

50. Sav arino E, Mei F, Parodi A, et al. Gastrointestinal motility disor der assessment in system ic sclerosis. Rheum atology (Oxford) 2013;52:1095-100. Full Text

51. DeSouza NM, Williams AD, Wilson HJ, Gilderdale DJ, Coutts GA, Black CM. Fecal incontinence in scleroderma: assessment of the anal sphincter with thin-section endoanal MR imaging. Radiology 1998;208:529-35. Full Text

52. Parodi A, Sessarego M, Greco A, et al. Small intestinal bacterial ov ergrowth in patients suffering from scleroderma: clinical effectiveness of its er adication. Am J Gastroenterol 2008;103:1257-62. Full Text

53. Nikou GC, Toumpanakis C, Katsiari C, Charalambopoulos D, Sfikakis PP. Treatment of small intestinal disease in systemic sclerosis with octreotide: a prospective study in seven patients. J Clin Rheumatol 2007;13:119-23. Full Text

54. Burr SK, Alam A, Cohen R, Krogh K, Buntzen S, Em manuel A. Lack of effect of sacral nervestimulation for incontinence in patients with systemic sclerosis. Color ectal Dis 2 015;17:903 -7. Full Text

55. Mecoli C, Purohit S, Sandorfi N, Derk CT. Mortality, recurrence, and hospital course of patients with sy stemic sclerosis-related acute intestinal pseu doobstruction. J Rheumatol 2 014;41:2049-54. Full Text

56. Bharadwaj S, Tandon P, Gohel T, et al. Gastrointestinal manifestations, malnutrition, and role of enteral and parenteral nutrition in patients with scleroderma. J Clin Gastroenterol 2015;49:559-64. Full Text

57. Frech TM, Khanna D, Maranian P, Frech EJ, Sawitzke AD, Murtaugh MA. Probiotics for the treatment of sy stemic scler osis-associated gastrointestinalbloating/distention. Clin ExpRheumatol 2011;29(Suppl 65):S22-5. 
58. Papachristos DA, Nikpour M, Hair C, Stevens WM. Intravenous cy clophosphamide as a therapeutic option for sev ere refractory gastric antral vascular ectasia in sy stemic sclerosis. Intern Med J 2015; 45:1077-81. Full Text

59. Schulz SW, O'Brien M, Maqsood M, Sandorfi N, Del Galdo F, Jimenez SA. Improvement of severe sy stemic sclerosis-a ssociated gastric a ntral vascular ectasia following immun osuppressive treatment with intraven ous cycloph osphamide. J Rheumatol 2009; 36:1653-6. Full Text 6o. Lor enzi AR, Johnson AH, Davies G, GoughA. Gastric antralvascular ectasia in systemic sclerosis: complete resolution with m ethylprednisolone and cy clophosphamide. Ann Rheum Dis 2001;60:796-8. Full Text

61. Raja J, Nihtyanova SI, Murray CD, Denton CP, Ong VH. Sustain ed ben efit from intraven ou im munoglobulin therapy for gastrointestinal involvement in sy stemic sclerosis. Rheumatology (Oxford) 2016 ; 55:115-19. Full Text 